\title{
Searching in the immune checkpoint black box
}

To identify
genes
potentially
involved
in evading
the tumour
response, the
authors used
CRISPR-
Cas9 to
systematically
knock out
2,368 genes
expressed
by B16
melanoma
cells

Checkpoint blockade through inhibition of programmed cell death protein 1 (PD1), which enables tumours to evade the immune system, has radically changed the treatment and prognosis of many cancers, especially melanoma. However, a majority of patients do not respond to PD1 inhibition, and great effort is now invested in identifying new drugs that, when used in combination with PD1 inhibitors, can increase the response to this treatment. Reporting in Nature, the team led by Haining has identified that deletion of Ptpn2, among other genes, in tumour cells makes them more susceptible to PD1 inhibitors.

To identify genes potentially involved in evading the tumour response, the authors used CRISPR-Cas9 to systematically knock out 2,368 genes expressed by B16 melanoma cells. Then, they transplanted the tumour cells into

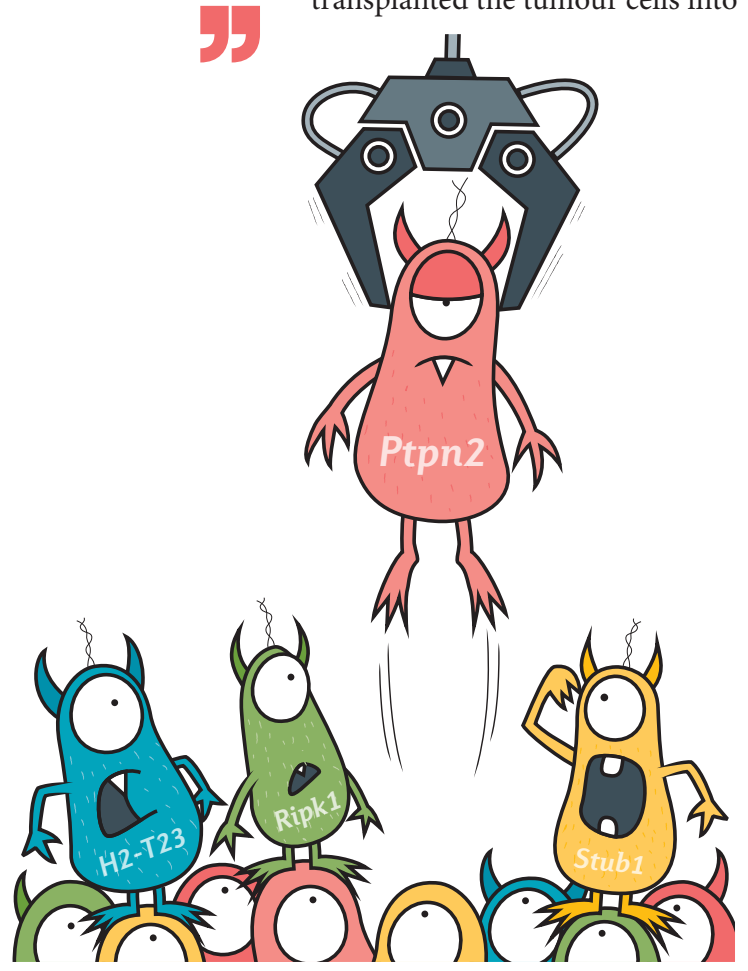

Neil Smith/Macmillan Publishers Limited
$\mathrm{Tcra}^{-/-}$mice (which lack CD4 ${ }^{+}$and $\mathrm{CD}^{+} \mathrm{T}$ cells and cannot develop an adaptive immune response) as controls and into wild-type mice that were subsequently treated with a granulocyte-macrophage colony-stimulating factor

(GM-CSF)-secreting tumour cell vaccine (GVAX) or GVAX com-

bined with a monoclonal antibody against PD1.

Next, they analysed the tumours that had responded to the treatment and that were sensitive to PD1 blockade as a result of the missing target gene. Among genes already known to be involved in immune evasion, such as PD1 ligand 1 (PDL1) and CD47, the authors identified 50 other genes whose deletion conferred sensitivity to PD1 inhibition. They selected four of these genes - Ptpn2, a phosphatase involved in signalling processes; $H 2-T 23$, which encodes QA1B (HLA-E in humans), a protein that binds the inhibitory receptor NKG2A on T cells and natural killer cells; Ripk1, a kinase that regulates cell death and inflammation; and Stub1, an E3 ubiquitin ligase that is involved in the regulation of the unfolded protein response - based on their highest cumulative score for further validation. Deletion of each of the four genes strongly inhibited tumour cell growth in wild-type animals treated with PD1 inhibitors, but these cells grew at equivalent rates to control tumour cells in vitro and in $\mathrm{Tcra}^{-1-}$ mice. Treatment with PD1 inhibitors cured all the animals with H2-T23-null B16 tumours, whereas it eradicated only 1 out of 10 tumours in control mice.

Similarly, loss of Ptpn2 also sensitized tumours to immunotherapy in vivo. Ptpn2-null B16 tumours were significantly more sensitive to PD1 inhibition
- but did not show any growth disadvantage in the absence of T cell-mediated immunity or PD1 inhibition. Overexpression of Ptpn2 in control B16 tumour cells led to an outgrowth of tumour cells in mice treated with PD1 inhibitors, suggesting that increased PTPN2 expression might confer resistance to immunotherapy, although analysis of PTPN2 expression in patients who were resistant to treatment with immune checkpoint inhibitors was inconclusive.

How does loss of Ptpn 2 enhance the efficacy of immunotherapy? Further experiments revealed that loss of Ptpn 2 in tumour cells increased antigen presentation and sensitivity to cytotoxic $\mathrm{CD}^{+} \mathrm{T}$ cells, and this was mediated by interferon- $\gamma$ (IFN $\gamma)$.

Finally, the authors also identified genes that, when deleted, were enriched in mice that had received immunotherapy, as these genes could be involved in mediating resistance to the treatment. They identified five genes required for sensing and signalling through the IFN $\gamma$ pathway (Stat1, Jak1, Jak2, Ifngr1 and Ifngr2). Indeed, tumours deficient in Stat 1 or Ifngr 1 grew significantly faster than wild-type tumours when treated with immunotherapy.

This study not only offers an obvious target in PTPN2 - even though the development of phosphatase inhibitors is notoriously challenging - but also offers a platform for identifying many more target genes that might mediate sensitivity and resistance to checkpoint inhibition.

M. Teresa Villanueva, Senior Editor Nature Reviews Drug Discovery This article originally appeared in Nature Rev. Drug Discovery (doi:10.1038/nrd.2017.163).

ORIGINAL ARTICLE Manguso, R. T. et al. In vivo

CRISPR screening identifies Ptpn2 as a cancer immunotherapy target. Nature 547, 413-418 (2017) 